\title{
An In Silico Approach to Enzymatic Synthesis of Fucooligosaccharides Using $\alpha$-L-Fucosidase from Thermotoga maritima ${ }^{\dagger}$
}

Emmanuel Pérez-Escalante 1, Luis Guillermo González-Olivares ${ }^{1}$, Araceli Castañeda-Ovando 1, Alma Elizabeth Cruz-Guerrero ${ }^{2}$, John F. Trant ${ }^{3}$, Wendolyne López-Orozco ${ }^{1}$, Luis Humberto Mendoza-Huizar 1,* and Sergio Alatorre-Santamaría 2,*

Citation: Pérez-Escalante, E.;

González-Olivares, L.G.;

Castañeda-Ovando, A.;

Cruz-Guerrero, A.E.; Trant, J.F.;

López-Orozco, W.;

Mendoza-Huizar, L.H.;

Alatorre-Santamaría, S. An In Silico

Approach to Enzymatic Synthesis of Fucooligosaccharides Using

$\alpha$-L-Fucosidase from Thermotoga

maritima. 2021, 3, 10

https://doi.org/10.3390

ecsoc-24-08303

Academic Editors: Julio A. Seijas and M. Pilar Vázquez-Tato

Published: 14 November 2020

Publisher's Note: MDPI stays neutral with regard to jurisdictional claims in published maps and institutional affiliations.

Copyright: (C) 2021 by the authors. Licensee MDPI, Basel, Switzerland. This article is an open access article distributed under the terms and conditions of the Creative Commons Attribution (CC BY) license (http://creativecommons.org/licenses /by/4.0/).
1 Área Académica de Química, Universidad Autónoma del Estado de Hidalgo, Carretera Pachuca-Tulancingo km 4.5 Mineral de la Reforma, Hidalgo 42184, Mexico; emmanuel_perez@uaeh.edu.mx (E.P.-E.); lgonzales@uaeh.edu.mx (L.G.G.-O.); ovandoa@uaeh.edu.mx (A.C.-O.); wen.lopez.orozco@gmail.com (W.L.-O.)

2 Departamento de Biotecnología, División de Ciencias Biológicas y de la Salud, Universidad Autónoma Metropolitana, Unidad Iztapalapa, Av. San Rafael Atlixco 186, Ciudad de Mexico 09340, Mexico; aec@xanum.uam.mx

3 Department of Chemistry and Biochemistry, University of Windsor, 401 Sunset Ave., Windsor, ON N9B 3P4, Canada; j.trant@uwindsor.ca

* Correspondence: hhuizar@uaeh.edu.mx (L.H.M.-H.); salatorre@xanum.uam.mx (S.A.-S.)

† Presented at the 24th International Electronic Conference on Synthetic Organic Chemistry, 15 November-15 December 2020; Available online: https:/ecsoc-24.sciforum.net/ (accessed on 14 November 2020).

Abstract: Fucooligosaccharides comprise the primary group of human milk oligosaccharides. Due to their beneficial properties, a series of synthetic methods have been proposed to obtain them. Enzymatic methods show great promise, and $\alpha$-L-fucosidase from Thermotoga maritima has emerged as a powerful catalyst for their production. Nonetheless, the enzyme's limited substrate scope has delayed its wider application. The present work aims to compare the relative reactivity of fucose, pNP-fucose, and ethyl-fucose, while also exploring the molecular interactions of these fucosyl-donors with the enzyme through a combination DFT and docking analysis. The HOMO-LUMO band gaps range from -7.14571 to $-4.24429 \mathrm{eV}$, with $\alpha / \beta$-pNP-fucose and $\alpha$-fucose being the three most reactive compounds. Moderate association energies between -6.4 to $-5.5 \mathrm{kcal} \cdot \mathrm{mol}^{-1}$ were found in the docking analysis, with $\alpha$-pNP-fucose and both anomers of ethyl-fucose demonstrating the poorest affinity. In the case of $\alpha / \beta$-lactose affinity to the $\beta$-fucose/enzyme complex, no significant differences were shown. We conclude that the best fucosyl-donors for transfucosylation are those that maintain an enzyme affinity and reactivity similar to $\mathrm{pNP}$-fucose.

Keywords: Fucooligosaccharides; $\alpha$-L-fucosidase; DFT study; molecular docking

\section{Introduction}

Fucooligosaccharides (FucOS) are the main oligosaccharides in human milk comprising $65-77 \%$ of the total oligosaccharide content [1]. Due to their antimicrobial, immunomodulatory, and prebiotic activities, as well as their promise to function as developmental cognitive enhancers, their incorporation into commercial formulations has become highly desirable $[1,2]$. As their isolation is complex due to their low abundance in animal milk, attention has turned to synthesis [1,2]. Fermentation is the most efficient, with generally recognize as safe (GRAS) certification from the U.S. Food and Drug Administration (FDA) permitting the addition of 2'-fucosyllactose ( 2 'FL) to infant formula [2]. Another synthetic alternative with recent promising results is the use of isolated enzyme, which 
requires the yield optimization of FucOS by fucosyl-transferases. Unfortunately, this approach presents the inconvenience of requiring nucleotide sugars as fucosyl-donors, which are more expensive than those used for fucosidases [1,3]. Consequently, FucOS synthesis by fucosyl-hydrolases like the $\alpha$-L-fucosidase from Thermotoga maritima has gained importance, as this pathway allows the use either of less expensive fucosyl-donors or even agro-industrial waste [3-8]. However, this enzymatic route provides lower yields than the transferase, or involves the release of toxic compounds such as $p$-nitrophenol [5-8]. Alternatives are highly desirable. Thus, the present work aimed to determine the relative reactivity of three non-classical fucosyl-donors through an in silico study to propose substrate alternatives for the enzymatic synthesis of FucOS.

\section{Methods}

\subsection{Geometry Optimization and HOMO-LUMO Parameters}

All compounds were totally geometry optimized through the Density Functional Theory (DFT) with the B3LYP/6-311++G(2d,2p) basis set using water as solvent. The highest occupied molecular orbital (HOMO) and lowest unoccupied molecular orbital (LUMO) density surfaces were visualized with Gabedit 2.5.0. [9].

\subsection{Molecular Docking for Hydrolysis and Transfucosylation Process}

The A chain of the $\alpha$-L-fucosidase crystal from Thermotoga maritima (PDB: 1ODU) was prepared with the DockPrep tool implemented in Chimera 1.13.1 [10], prior to its use as the receptor for molecular docking. All molecular dockings were performed by Autodock VINA [11] through the PyRx software [12], taking as reference the amino acids from the active site cited by Sulzenbacher et al. [13], with coordinates for the search space centered on x: -20.63 , y: 19.03, and z: 63.32, with a grid cube with dimensions of $25.00 \AA$. In the case of the hydrolysis, a single docking step was performed for each fucosyl-donor and the receptor. Meanwhile for the transfucosylation process, a sequential docking sequence was employed. First, $\beta$-fucose was docked to the enzyme in order to form the pre-complex, then docking was performed again with lactose. The best binding mode for each interaction was obtained and its interactions were processed with the BIOVIA Discovery Studio Visualizer@ v19.1.0.18287 [14].

\section{Results and Discussion}

\subsection{HOMO-LUMO Parameters}

The HOMO-LUMO frontier orbitals and the bandgaps were calculated for both anomers of fucose, ethyl-fucose, $p$-nitrophenyl (pNP)-fucose, and lactose (Figure 1). In general, $\beta$-anomers showed a lower bandgap compared with the $\alpha$-anomers, except for the pNP-fucose anomers, which showed similar bandgaps. As the magnitude of the HOMO-LUMO gap directly relates to chemical reactivity, where larger bandgaps predict lower reactivity $[15,16]$, we predict that the $\beta$-anomers of fucose, ethyl-fucose, and lactose should prove less reactive than their $\alpha$-anomers; in contrast, both anomers of pNP-fucose should demonstrate similar reactivity. Ranking the series, $\alpha / \beta$-pNP-fucose with its electron withdrawing nature, unsurprisingly, is predicted to be the most reactive followed by $\alpha$-fucose. In general, the $\alpha$-anomers promise greater reactivity than the $\beta$ anomers (again, with the exception of the hot pNP electrophile) based on the distribution of density in the frontier molecular orbitals. 


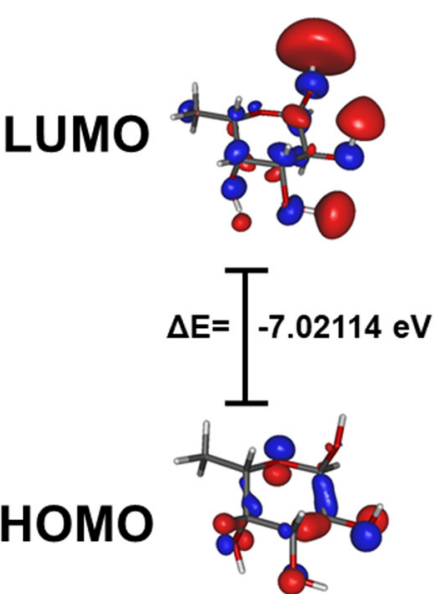

a)

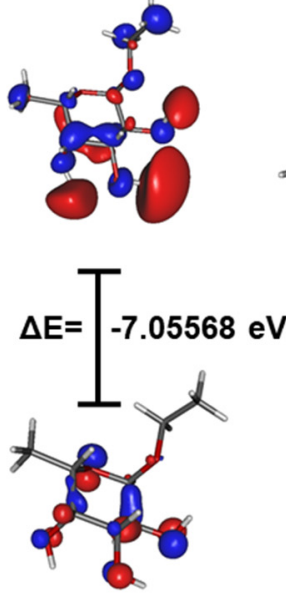

b)

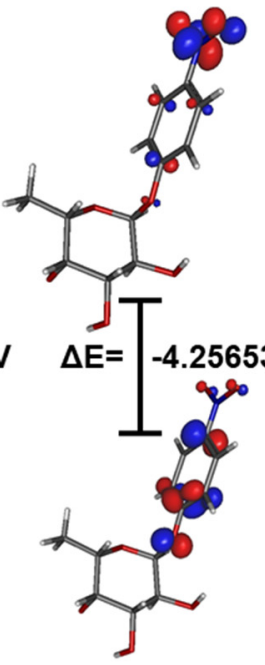

c)

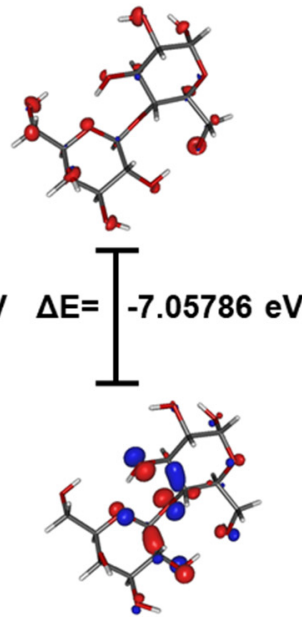

d)

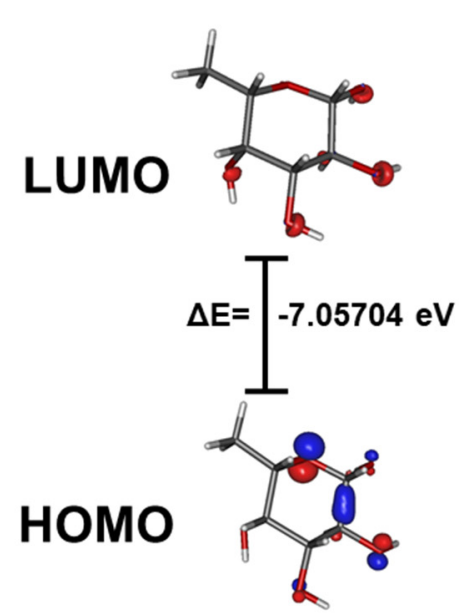

e)
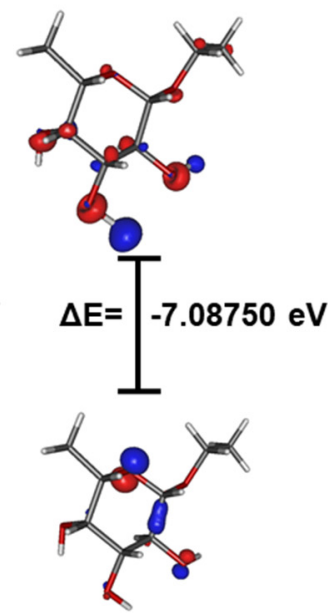

f)
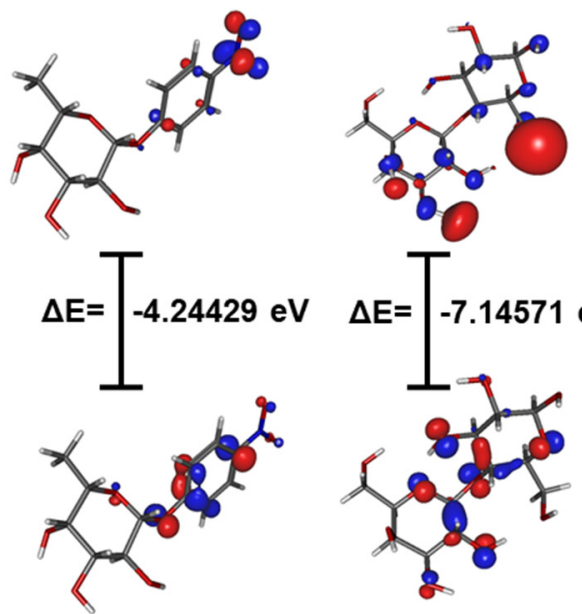

g)

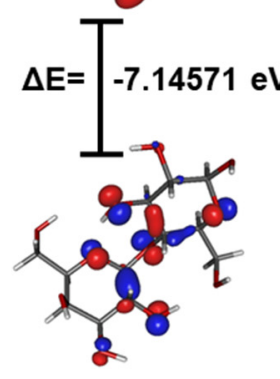

h)

Figure 1. HOMO and LUMO surfaces (blue: positive and red: negative) of (a) $\alpha$-fucose, (b) $\alpha$-ethyl-fucose, (c) $\alpha$-pNP-fucose, (d) $\alpha$-lactose, (e) $\beta$-fucose, (f) $\beta$-ethyl-fucose, (g) $\beta$-pNP-fucose, and (h) $\beta$-lactose, as well as their HOMO-LUMO band gap.

\subsection{Molecular Docking for Hydrolysis Processes}

Single molecular docking simulated the hydrolysis catalyzed by T. maritima's $\alpha$-L-fucosidase. Docking scores varied between -6.4 and $-5.5 \mathrm{kcal} \cdot \mathrm{mol}^{-1}$, with $\beta$-pNP-fucose showing the best affinity to the receptor, while $\alpha$-ethyl-fucose presented the worst (Table 1). A lower substrate-enzyme affinity could be correlated with lower complex stability and a tendency to destroy it [17]. Thus, among the six different tested molecules, $\alpha$-ethyl-fucose, $\beta$-ethyl-fucose, and $\alpha$-pNP-fucose appear to be the fucosyl donors most readily hydrolyzed once bound to the enzyme. 
Table 1. Coupling energies obtained for each fucosyl-donor and the $\alpha$-L-fucosidase from Thermotoga maritima.

\begin{tabular}{cc}
\hline Fucosyl-Donor & Coupling Energy $\left(\mathbf{k c a l} \cdot \mathbf{m o l}^{-1}\right)$ \\
\hline$\alpha$-fucose & -6.0 \\
$\beta$-fucose & -6.3 \\
$\alpha$-ethyl-fucose & -5.5 \\
$\beta$-ethyl-fucose & -5.8 \\
$\alpha$-pNP-fucose & -5.9 \\
$\beta$-pNP-fucose & -6.4 \\
\hline
\end{tabular}

The key non-covalent interactions were identified (Figure 2). Those identified for $\beta$-fucose/enzyme docking (Figure 2d), agree with those reported by Sulzenbacher et al. [13]. All showed essential $\pi$-interactions between the C -5 methyl group and aromatic residues on the enzyme. The last interactions propitiate that the sugar ring takes a perpendicular position to this hydrophobic region, favoring van der Waals interactions observed with the rest of the molecule sites [13]. An important change was found for the interaction with the Asp224, the amino acid responsible for the nucleophilic attack that forms the covalent glycosyl-intermediate [13]. This pre-reaction interaction is a hydrogen-bond for $\beta$-fucose; but is van der Waals type for $\alpha$-fucose and $\alpha / \beta$-pNP-fucose; is an unfavorable repulsive interaction for $\alpha$-ethyl-fucose; and a hydrogen bond with the $\mathrm{C}-4 \mathrm{OH}$ for $\beta$-ethyl-fucose, indicating the different binding modes of these two substrates. These insights could explain the differential docking energies and suggests that hydrolysis would prove more challenging.

a)

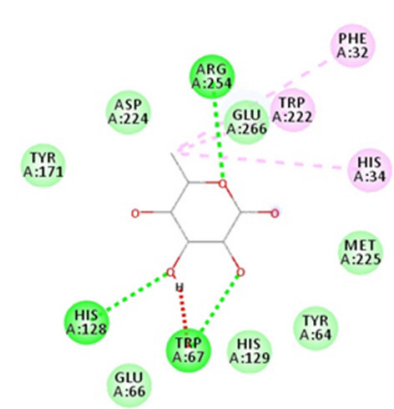

d)

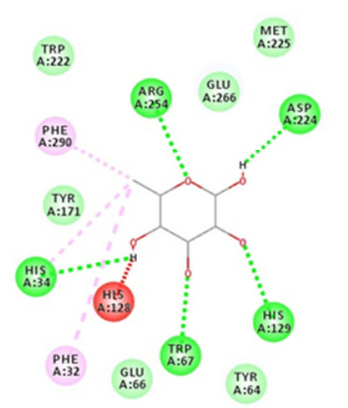

b)

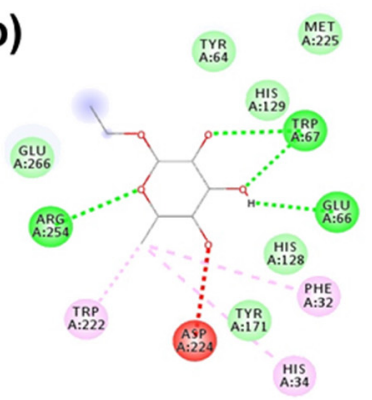

e)

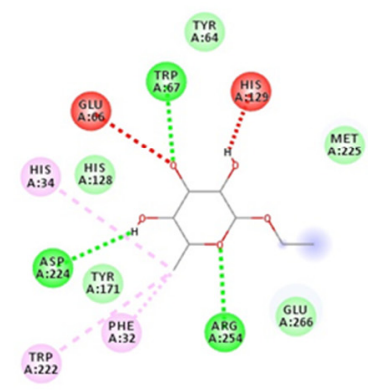

c)
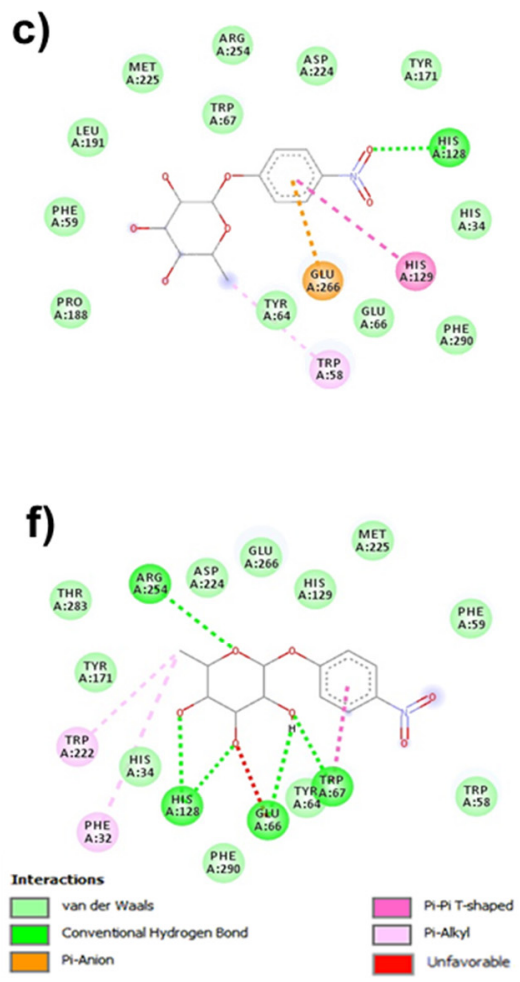

Figure 2. Mappings in 2D of the binding interactions among fucosyl-donors and the $\alpha$-L-fucosidase from Thermotoga maritima: (a) $\alpha$-fucose, (b) $\alpha$-ethyl-fucose, (c) $\alpha$-pNP-fucose, (d) $\beta$-fucose, (e) $\beta$-ethyl-fucose, and (f) $\beta$-pNP-fucose.

\subsection{Molecular Docking for Studying Transfucosylation}

In order to simulate a transfucosylation process, a sequential docking was performed. First, the $\beta$-fucose/enzyme complex was established by a single docking. The 
results were consistent with the reports of Sulzenbacher et al. [13], with $\alpha$ - or $\beta$-lactose, providing docking scores of -5.7 and $-5.8 \mathrm{kcal} \cdot \mathrm{mol}^{-1}$, respectively. However, the conformation was different for each (Figure 3). While $\alpha$-lactose adopts a position near the $\beta$-fucose binding site, $\beta$-lactose adheres preferentially to a more distal site. $\beta$-lactose has only a series of weak van der Waals interactions holding it in place, including with the key Glu266 residue, while $\alpha$-lactose forms strong hydrogen bonds with both Glu66 and Glu266, the amino acids responsible for the activation of acceptor groups for transfucosylation [13]. This suggests that the alpha anomer should be far more reactive, and indicates that mutarotation likely forms this anomer prior to transformation.

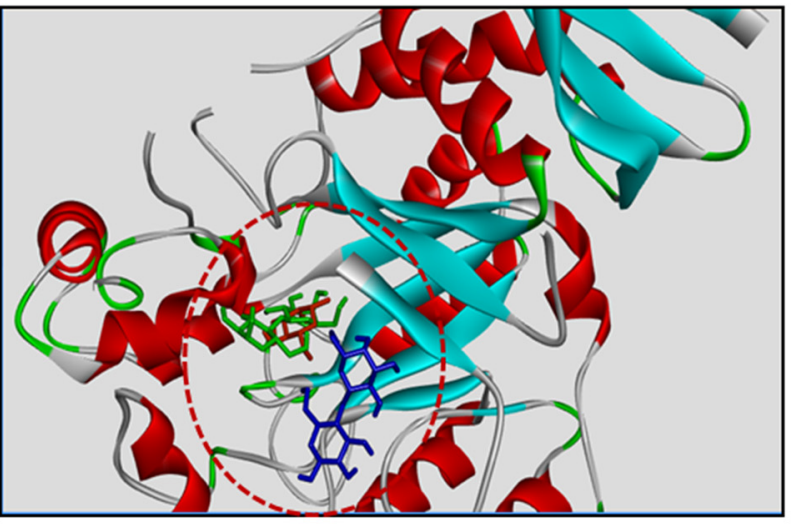

a)
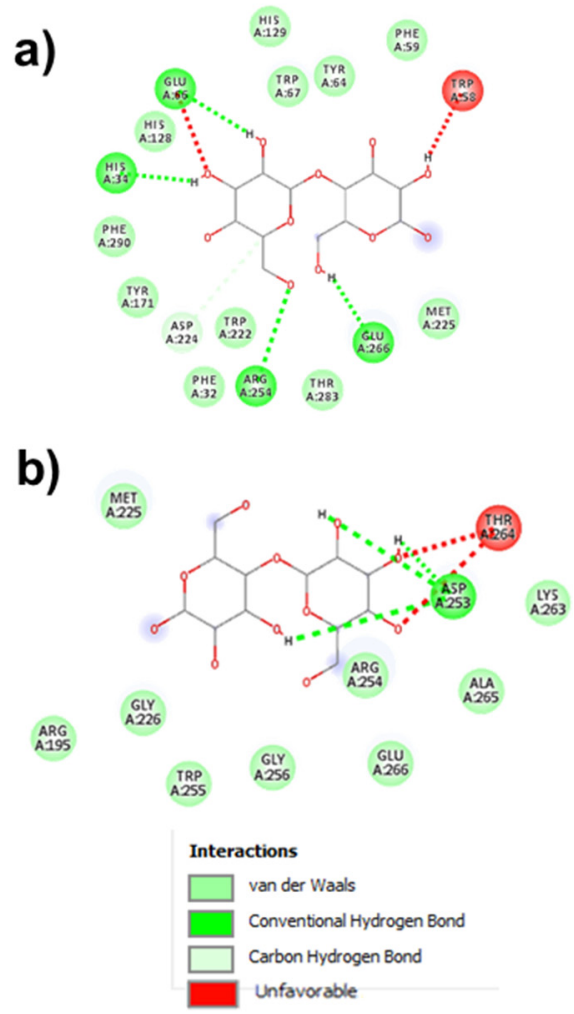

Figure 3. Binding position of $\alpha$-lactose (green colored) and $\beta$-lactose (blue colored) in the complex $\beta$-fucose (orange colored)/enzyme, as well as the molecular interactions of (a) $\alpha$-lactose and (b) $\beta$-lactose to the receptor.

On the other hand, the effect of the fucosyl-donors on the transfucosylation process can be related to the reactivity showed in the HOMO-LUMO gap, because previous in vitro studies have shown the effective transference of pNP-fucose to lactose to synthesize FucOS [5,6], while other studies have found low yields or long process when fucose itself is used as the donor $[7,8]$. Thus, ethyl-fucose could show similar results to those obtained with fucose mainly due to both molecules showing similar reactivity. Finally, according to in vitro results obtained earlier and the in silico insights found here, it is possible to hypothesize that fucosyl-donors with similar structure and/or reactivity to that of pNP-fucose could act as good substrates for transfucosylation with the $\alpha$-L-fucosidase from Thermotoga maritima.

\section{Conclusions}

In silico insights of the reactivity and molecular interactions with the $\alpha$-L-fucosidase from Thermotoga maritima obtained for each fucosyl-donor and in vitro results from literature allowed us to conclude that the best fucosyl-donors for transfucosylation reaction will be those with similar reactivity to pNP-fucose, so that the synthesis of compounds 
with similar structures, but lower toxicity, should be prioritized to find next generation fucsosyl transfer agents.

Author Contributions: Conceptualization, E.P.-E., L.G.G.-O. and S.A.-S.; methodology, E.P.-E. and L.H.M.-H.; software, L.H.M.-H. and W.L.-O.; validation, A.E.C.-G., J.F.T. and A.C.-O.; formal analysis, A.E.C.-G. and L.G.G.-O.; investigation, E.P.-E. and L.H.M.-H.; resources, L.H.M.-H., L.G.G.-O. and S.A.-S.; data curation, J.F.T. and A.C.-O.; writing - original draft preparation, E.P.-E.; writing - review and editing, S.A.-S. and J.F.T.; visualization, L.G.G.-O. and S.A.-S.; supervision, L.H.M.-H. and S.A.-S.; project administration, L.G.G.-O. and S.A.-S.; funding acquisition, L.H.M.-H., L.G.G.-O. and S.A.-S. All authors have read and agreed to the published version of the manuscript.

Institutional Review Board Statement: Not applicable.

Informed Consent Statement: Not applicable.

Data Availability Statement: The data presented in this study are available within the article.

Acknowledgments: E.P.-E. would like to acknowledge the Consejo Nacional de Ciencia y Tecnología (CONACYT) for granting the scholarship number 473399.

Conflicts of Interest: The authors declare no conflict of interest.

\section{References}

1. Pérez-Escalante, E.; Alatorre-Santamaría, S.; Castañeda-Ovando, A.; Salazar-Pereda, V.; Bautista-Ávila, M.; Cruz-Guerrero, A.E.; Flores-Aguilar, J.F.; González-Olivares, L.G. Human milk oligosaccharides as bioactive compounds in infant formula: Recent advances and trends in synthetic methods. Crit. Rev. Food Sci. Nutr. 2020, 1-34.

2. Bych, K.; Mikš, M.H.; Johanson, T.; Hederos, M.J.; Vigsnæs, L.K.; Becker, P. Production of HMOs using microbial hosts-From cell engineering to large scale production. Curr. Opin. Biotechnol. 2019, 56, 130-137.

3. Zeuner, B.; Meyer, A.S. Enzymatic transfucosylation for synthesis of human milk oligosaccharides. Carbohydr. Res. 2020, 493, 108029.

4. Wan, L.; Zhu, Y.; Zhang, W.; Mu, W. $\alpha$-L-Fucosidases and their applications for the production of fucosylated human milk oligosaccharides. Appl. Microbiol. Biotechnol. 2020, 104, 5619-5631.

5. Guzmán-Rodríguez, F.; Alatorre-Santamaría, S.; Gómez-Ruiz, L.; Rodríguez-Serrano, G.; García-Garibay, M.; Cruz-Guerrero, A. Synthesis of a fucosylated trisaccharide via transglycosylation by $\alpha$-L-fucosidase from Thermotoga maritima. Appl. Biochem. Biotechnol. 2018, 186, 681-691.

6. Shi, R.; Ma, J.; Yan, Q.; Yang, S.; Fan, Z.; Jiang, Z. Biochemical characterization of a novel $\alpha$-L-fucosidase from Pedobacter sp. and its application in synthesis of 3'-fucosyllactose and 2'-fucosyllactose. Appl. Microbiol. Biotechnol. 2020, 104, 5813-5826.

7. Usvalampi, A.; Medrano, M.R.; Maaheimo, H.; Salminen, H.; Tossavainen, O.; Frey, A.D. Production and characterization of Aspergillus niger GH29 family $\alpha$-fucosidase and production of a novel non-reducing 1-fucosyllactose. Glycoconj. J. 2020, 37, 221-229.

8. Zeuner, B.; Muschiol, J.; Holck, J.; Lezyk, M.; Gedde, M.R.; Jers, C.; Mikkelsen, J.D.; Meyer, A.S. Substrate specificity and transfucosylation activity of GH29 $\alpha$-L-fucosidases for enzymatic production of human milk oligosaccharides. New Biotechnol. 2018, 41, 34-45.

9. Allouche, A.R. Gabedit-A graphical user interface for computational chemistry softwares. J. Comput. Chem. 2011, 32, 174-182.

10. Pettersen, E.F.; Goddard, T.D.; Huang, C.C.; Couch, G.S.; Greenblatt, D.M.; Meng, E.C.; Ferrin, T.E. UCSF Chimera-A visualization system for exploratory research and analysis. J. Comput. Chem. 2004, 13, 1605-1612.

11. Trott, O.; Olson, A.J. AutoDock Vina: Improving the speed and accuracy of docking with a new scoring function, efficient optimization, and multithreading. J. Comput. Chem. 2010, 31, 455-461.

12. Dallakyan, S.; Olson, A.J. Small-molecule library screening by docking with PyRx. Methods Mol. Biol. 2015, 1263, $243-250$.

13. Sulzenbacher, G.; Bignon, C.; Nishimura, T.; Tarling, C.A.; Withers, S.G.; Henrissat, B.; Bourne, Y. Crystal Structure of Thermotoga maritima $\alpha$-L-Fucosidase insights into the catalytic mechanism and the molecular basis for fucosidosis. J. Biol. Chem. 2004, 279, 13119-13128.

14. Dassault Systèmes BIOVIA. Discovery Studio Visualizer; v19.1.0.18287; Dassault Systèmes: San Diego, CA, USA, 2019.

15. Talmaciu, M.M.; Bodoki, E.; Oprean, R. Global chemical reactivity parameters for several chiral beta-blockers from the Density Functional Theory viewpoint. Clujul Med. 2016, 89, 513-518.

16. Boukli-Hacene, F.; Merad, M.; Ghalem, S.; Soufi, W. DFT Study of the Interaction of Cu (II), Zn (II), Sn (II) with Carbohydrates in Aqueous Solution. J. Chem. 2014, 8, 1009-1017.

17. Bettelheim, F.A.; Brown, W.H.; Campbell, M.K.; Farrell, S.O. Introduction to Organic and Biochemistry, 7th ed.; Cengage Learning: Belmont, CA, USA, 2010; pp. 353-355. 